\title{
Macrophage Migration Inhibitory Factor (MIF) Enzymatic Activity and Lung Cancer
}

\author{
Leona Mawhinney, ${ }^{1}$ Michelle E Armstrong, ${ }_{1}^{1}$ Ciaran $O^{\prime}$ Reilly, ${ }^{2}$ Richard Bucala, ${ }^{3}$ Lin Leng, ${ }^{3}$ \\ Gunter Fingerle-Rowson, ${ }^{4}$ Darren Fayne, ${ }^{2}$ Michael P Keane, ${ }_{1}^{1}$ Aisling Tynan, ${ }^{1}$ Lewena Maher, ${ }_{1}^{1}$ \\ Gordon Cooke, ${ }^{1}$ David Lloyd, ${ }^{5}$ Helen Conroy, ${ }^{1}$ and Seamas C Donnelly ${ }^{1}$
}

${ }^{1}$ Conway Institute for Biomolecular and Biomedical Research, School of Medicine and Medical Science, University College Dublin, Dublin, Ireland; ${ }^{2}$ Molecular Design Group, School of Biochemistry and Immunology, Trinity Biomedical Sciences Institute, Trinity College Dublin, Dublin, Ireland; ${ }^{3}$ Department of Internal Medicine, Yale University School of Medicine, New Haven, Connecticut, United States of America; ${ }^{4}$ Department of Internal Medicine 1, University Hospital Cologne, Centre for Integrated Oncology KölnBonn, Cologne, Germany; and ${ }^{5}$ Division of Health Sciences, University of South Australia, Adelaide, Australia

\begin{abstract}
The cytokine macrophage migration inhibitory factor (MIF) possesses unique tautomerase enzymatic activity, which contributes to the biological functional activity of MIF. In this study, we investigated the effects of blocking the hydrophobic active site of the tautomerase activity of MIF in the pathogenesis of lung cancer. To address this, we initially established a Lewis lung carcinoma (LLC) murine model in Mif-KO and wild-type (WT) mice and compared tumor growth in a knock-in mouse model expressing a mutant MIF lacking enzymatic activity (Mif ${ }^{P I G}$ ). Primary tumor growth was significantly attenuated in both Mif-KO and Mif ${ }^{P I G}$ mice compared with WT mice. We subsequently undertook a structure-based, virtual screen to identify putative small molecular weight inhibitors specific for the tautomerase enzymatic active site of MIF. From primary and secondary screens, the inhibitor SCD-19 was identified, which significantly attenuated the tautomerase enzymatic activity of MIF in vitro and in biological functional screens. In the LLC murine model, SCD-19, given intraperitoneally at the time of tumor inoculation, was found to significantly reduce primary tumor volume by $90 \%(p<0.001)$ compared with the control treatment. To better replicate the human disease scenario, SCD-19 was given when the tumor was palpable (at d 7 after tumor inoculation) and, again, treatment was found to significantly reduce tumor volume by $81 \%(p<0.001)$ compared with the control treatment. In this report, we identify a novel inhibitor that blocks the hydrophobic pocket of MIF, which houses its specific tautomerase enzymatic activity, and demonstrate that targeting this unique active site significantly attenuates lung cancer growth in in vitro and in vivo systems.
\end{abstract}

Online address: http://www.molmed.org

doi: $10.2119 / \mathrm{molmed} .2014 .00136$

\section{INTRODUCTION}

Lung cancer is the leading cause of cancer deaths worldwide (1). It is estimated that the 5-year survival rate is at most $16 \%$ (2). Therefore, novel therapeutic targets for lung cancer are urgently required.

Macrophage migration inhibitory factor (MIF) is a proinflammatory cytokine that has been implicated in the pathogenesis of chronic inflammatory diseases (3-6). MIF is expressed by a number of cell types such as macrophage cells (7), lymphocytes (8), neutrophils (9) and eosinophils (4). High levels of preformed MIF, as well as MIF mRNA, were previously found in unstimulated macrophages in the RAW 264.7 cell line (7). In

Address correspondence to Leona Mawhinney, School of Medicine, Trinity Biomedical Science Institute, Trinity College Dublin, Dublin 2, Ireland. Phone: +353-(0) 7-896-4435; E-mail: mawhinnl@tcd.ie.

Submitted July 10, 2014; Accepted for publication December 17, 2014; Epub (www.molmed.org) ahead of print December 17, 2014.

\section{The Feinstein Institute for Medical Research} Empowering Imagination. Pioneering Discovery.

addition, an expanding body of recent evidence has highlighted the important role for this proinflammatory cytokine in cancer $(10,11)$. MIF is expressed by a variety of cancers including prostate (12), colon (13), liver (14) and lung (15), and a number of protumor functions have been assigned to this protein. These functions include the downregulation of the tumor suppressor p53 and prevention of p53induced apoptosis by MIF, thereby facilitating malignant transformation (16). MIF also promotes constitutive extracellular signal-regulated kinase (ERK) activation mirroring the actions of oncogenes such as RAS. MIF has been found to support hypoxic adaptation of cells by inducing stabilization of hypoxiainducible factor 1- $\alpha$ (HIF1 $\alpha$ ) (17). MIF readily contributes to a microenviron- 
ment favoring tumor growth and proliferation by promoting angiogenesis required to sustain tumor growth.

Specific studies in lung cancer have identified MIF as a key regulator of tumor growth. MIF expression has been shown to correlate with expression of angiogenic chemokines in non-small cell lung cancer (18). Specific knockdown of MIF expression or inhibition of its function significantly reduced migration and invasion of lung adenocarcinoma cells (19). In a model of chronic lung injury, enhanced MIF expression promoted lung tumor growth, highlighting the key role of this cytokine in tumor development in chronic inflammatory diseases (20). These studies provide persuasive evidence that MIF is a valid therapeutic target in lung cancer.

A unique functional characteristic for this cytokine is that it possesses enzymatic activity. Specifically, it has the ability to catalyze the tautomerization of the nonphysiological substrate D-dopachrome into an indole derivative (21). To date, the precise role of the tautomerase enzymatic activity of MIF in clinical disease has not been clearly defined. However, with the development of specific inhibitors, this enzymatic activity has been shown to be critical for protein function in a variety of diseases from inflammation to cancer (22-26), most likely by virtue of structural features of the active site mediating critical protein: protein interactions (21). On the basis of this expanding body of evidence, we developed specific small-molecular-weight inhibitors targeting the tautomerase enzymatic activity of MIF as a potential therapeutic strategy in lung cancer.

Here, we report the characterization of a novel inhibitor of MIF tautomerase activity, namely SCD-19. Subsequently, we show that this inhibitor has the capacity to significantly attenuate lung cancer growth in in vitro and in vivo systems.

\section{MATERIALS AND METHODS}

\section{Structure-Based Drug Design}

FRED (version 2.6; OpenEye Scientific Software, Sante Fe, NM, USA; www.eyesopen.com), a protein-ligand based docking program, was used to conduct a virtual screening campaign against the tautomerase active site of MIF. The crystal structure, 1CA7, was downloaded from pdb.org (Research Collaboratory for Structural Bioinfomatics Protein Data Bank [RCSB PDB]) to evaluate the programs ability to reproduce accurate bioactive conformations. The hydroxyphenyl pyruvate ligand was removed from the active site, conformations were generated by using OMEGA (OpenEye Scientific Software) and the new library was docked into the tautomerase active site. Analysis of the various scoring functions available in FRED showed that the top-ranked pose in the Chemgauss 3 scoring function had the lowest root-mean-square deviation (RMSD) when compared with the crystal structure ligand. A training data set was also generated consisting of 40 known MIF actives and 960 putative decoy compounds. Conformers of each ligand were enumerated and docked into the tautomerase active site by using Chemgauss3. The ability of FRED to distinguish the actives from the decoys was assessed through ROC curve analysis. With the docking protocols validated, a vendor data set consisting of approximately 205,000 molecules was downloaded from SPECS.net and screened. Compounds were chosen on the basis of their Chemgauss 3 score. These compounds then were assessed in in vitro cellular systems with regard to their efficacy in inhibiting MIF biological activity.

\section{Cells and Reagents}

The RAW 264.7 cell line (European Collection of Cell Cultures [ECACC], a Culture Collection of Public Health England, Salisbury, UK) and Lewis lung carcinoma (LLC) cell line (Tariq Sethi, Kings College London) were cultured in Dulbecco modified Eagle medium (DMEM) containing 10\% FCS, $2 \mathrm{mmol} / \mathrm{L}$ L-glutamine, 10,000 IU penicillin and 10,000 $\mu \mathrm{g} / \mathrm{mL}$ streptomycin (Gibco [Thermo Fisher Scientific, Waltham, MA, USA]). Cells were maintained in sterile environment at $37^{\circ} \mathrm{C}$ in humidified and concentrated $\mathrm{CO}_{2}(5 \%)$ atmosphere. All cells were subcultured on reaching 80-90\% confluency.

\section{MIF Tautomerase Assay}

L-Dopachrome substrate was prepared through oxidation of L-dopachrome methyl ester with sodium periodate. ISO-1 represents the most characterized inhibitor of the enzymatic activity of MIF. Recombinant MIF (donated by Richard Bucala, Yale University) (100 ng/mL) was incubated with substrate along with SCD-19 or control ISO1 at the indicated final concentrations. The decrease in absorbance was measured at $475 \mathrm{~nm}$ every $10 \mathrm{~s}$ for $10 \mathrm{~min}$.

\section{Cellular Growth Rate, Cytokine Analysis and Cell Death Assays}

LLC cells were plated at a density of $5 \times 10^{4}$ cells $/ \mathrm{mL}$ in complete DMEM. After overnight adherence, $100 \mathrm{ng} / \mathrm{mL}$ recombinant macrophage migration inhibitory factor (rMIF) was added along with the inhibitors or dimethyl sulfoxide (DMSO) in serum-free medium. Cells were harvested after $48 \mathrm{~h}$ by trypsinization and counted by using a hemocytometer. RAW 264.7 macrophage cells were plated at $1 \times 10^{5} / \mathrm{mL}$ in complete DMEM. Sixteen hours later, cells were treated with ISO-1 or SCD-19 at a final concentration of 10,50 or $100 \mu \mathrm{mol} / \mathrm{L}$ for $30 \mathrm{~min}$ before stimulation with $100 \mathrm{ng} / \mathrm{mL}$ lipopolysaccharide (LPS) (Sigma-Aldrich, St. Louis, MO, USA). Supernatants were collected after $16 \mathrm{~h}$. Tumor necrosis factor (TNF) $\alpha$ concentrations were determined by using a $\mathrm{TNF} \alpha$ specific enzyme-linked immunosorbent assay (ELISA) (R\&D Systems, Abingdon, $\mathrm{UK})$. Levels of prostaglandin $\mathrm{E}_{2}\left(\mathrm{PGE}_{2}\right)$ were also assessed in the supernatants by ELISA (R\&D Systems). Cell death was determined by measuring $\mathrm{LDH}$ present in collected supernatant (Roche, Basel, Switzerland; see Supplementary Figure S1).

\section{Animal Experiments}

Specific pathogen-free female C57BL/ 6 mice at $6-8$ wks old ( $20 \mathrm{~g})$ were pur- 
chased from Charles River Laboratories (Ballina, Ireland). Mif-KO and Mif ${ }^{P 1 G}$ (21) genetically modified animals were bred in-house and used at 6-8 wks old. They were maintained in the Biomedical Facility at University College Dublin under laminar airflow conditions. All animals had free access to standard laboratory food and water. All housing was temperature controlled and had a 12-h light and dark cycle. This work was approved by the University College Dublin animal research committee and complies with the international best practice for the care and use of laboratory animals.

The LLC murine model represents a classic in vivo model of aggressive lung cancer. The model has been described previously (27). Briefly, log-phase cultures of LLC cells were harvested by trypsinization and washed three times with sterile phosphate-buffered saline (PBS). The cells were resuspended at a cell density of $2.5 \times 10^{6} / \mathrm{mL}$ in PBS. The $5 \times 10^{5}$ LLC cells were injected subcutaneously in a $200 \mu \mathrm{L}$ volume into the left flank of 6- to 8-wk-old C57BL/ 6 mice. Animals were monitored for the duration of the experiment, and tumor measurements were recorded twice weekly with digital calipers. Tumor volume $\left(\mathrm{mm}^{3}\right)$ was calculated as $0.5 \times L \times W^{2}$, where length $(L)$ is the larger tumor diameter and width $(W)$ is the smaller tumor diameter. Tautomerase inhibitors, dissolved in 5\% DMSO, were administered intraperitoneally twice weekly at a concentration of $35 \mathrm{mg} / \mathrm{kg}$ throughout the course of the experiment starting on $\mathrm{d} 0$ (prophylactic model) or when the tumor was palpable on the flank of the mouse (d 7) (therapeutic model).

\section{Statistical Analysis}

Data are expressed as mean \pm standard deviation (SD) from cumulative data, and the number of experiments performed is as outlined. The statistical difference between the groups was determined by using analysis of variance (ANOVA). Multiple comparisons between groups were then assessed by using the Bonferroni ad hoc test.
All supplementary materials are available online at www.molmed.org.

\section{RESULTS}

\section{Structure-Based Drug Design Identifies a Novel Inhibitor of MIF Tautomerase Activity}

MIF has been identified as a therapeutic target in autoimmune, inflammatory and malignant disease. We set out to identify novel inhibitors of MIF to be used in the treatment of lung cancer. By using a commercial database, we screened >200,000 compounds for their potential to interact with the active site of MIF. Compounds were ranked according to affinity, and those with the highest binding scores were assayed in vitro. Inhibition of L-dopachrome catalysis by recombinant MIF in the presence of the inhibitors was assessed. SCD-19 [3-(2'-methylphenyl)-isocoumarin] (Figure 1A) was identified as our most effective inhibitor of MIF. At a concentration of $100 \mu \mathrm{mol} / \mathrm{L}, \mathrm{SCD}-19$ was capable of complete inhibition of MIF tautomerase activity, whereas ISO-1 is also shown to be less effective at inhibiting the tautomerase activity of MIF (Figure 1B). Assessment of the levels of LDH in cell supernatants demonstrated that SCD-19 was nontoxic and did not induce cell death (see Supplementary Figure S1).

\section{SCD-19 Inhibits the LPS-Associated Release of $\mathrm{PGE}_{2}$ and TNF $\alpha$}

The tautomerase enzymatic activity of MIF has been implicated in a number of its molecular functions. Enzymatically active MIF promotes optimal release of inflammatory mediators such as $\mathrm{TNF} \alpha$ and $\mathrm{PGE}_{2}$ from LPS-stimulated cells. We examined the ability of SCD-19 to inhibit MIF in this context. LPS was used to stimulate the release of TNF $\alpha$ and $\mathrm{PGE}_{2}$ from RAW 264.7 macrophage cells. Cell supernatants were then analyzed by ELISA. SCD-19 and IS0-1 were shown to dose dependently reduce TNF $\alpha$, with SCD-19 being more effective at all concentrations (SCD-19
A

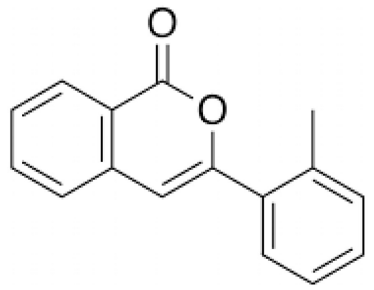

B

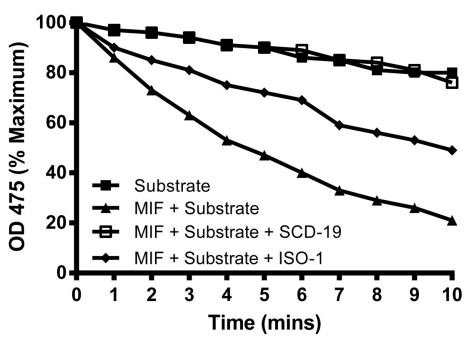

C

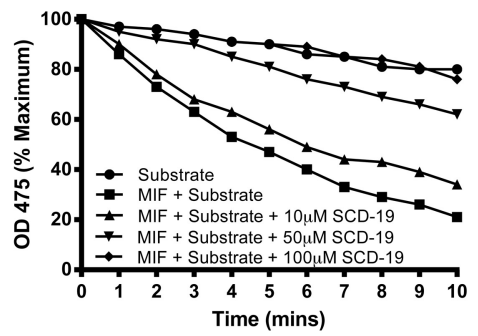

Figure 1. SCD-19 inhibits MIF enzymatic activity. (A) Structure of tautomerase inhibitor SCD-19. (B) Inhibition of MIF tautomerase activity by SCD-19 (100 $\mu \mathrm{mol} / \mathrm{L})$ and ISO-1 (100 $\mu \mathrm{mol} / \mathrm{L})$. Recombinant MIF was incubated with L-dopachrome methyl ester substrate with or without SCD-19 or ISO-1. Inhibition of tautomerase activity was assessed by measuring absorbance at $475 \mathrm{~nm}$ over $10 \mathrm{~min}$. (C) Inhibition of MIF tautomerase activity by increasing doses of SCD-19 (10, 50 or $100 \mu \mathrm{mol} / \mathrm{L})$. Recombinant MIF was incubated with L-dopachrome methyl ester substrate with or without SCD-19 at 10 , 50 or $100 \mu \mathrm{mol} / \mathrm{L}$. Inhibition of tautomerase activity was assessed by measuring absorbance at $475 \mathrm{~nm}$ over $10 \mathrm{~min}$.

[100 $\mu \mathrm{mol} / \mathrm{L}], 48 \%$ reduction \pm 3.44 , ${ }^{* * *} p<0.001$; ISO-1 [100 $\mu \mathrm{mol} / \mathrm{L}$ ], 36\% reduction \pm 5.52 ; Figures $2 \mathrm{~A}, \mathrm{~B})$. Similarly, $\mathrm{PGE}_{2}$ inhibition was seen to be more significant with SCD-19 compared with ISO-1 (SCD-19, 34\% reduction \pm 0.023 , ${ }^{* *} p<0.01$; ISO-1, 25\% reduction \pm 0.062 , ${ }^{*} p<0.05$ ) (Figure 2C). 
A

B

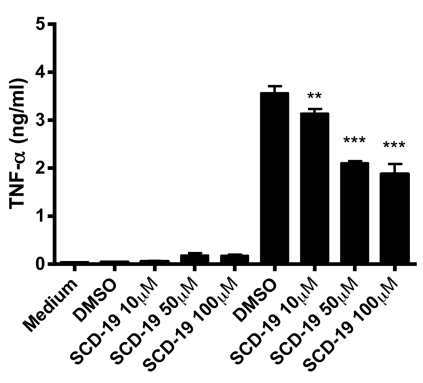

C
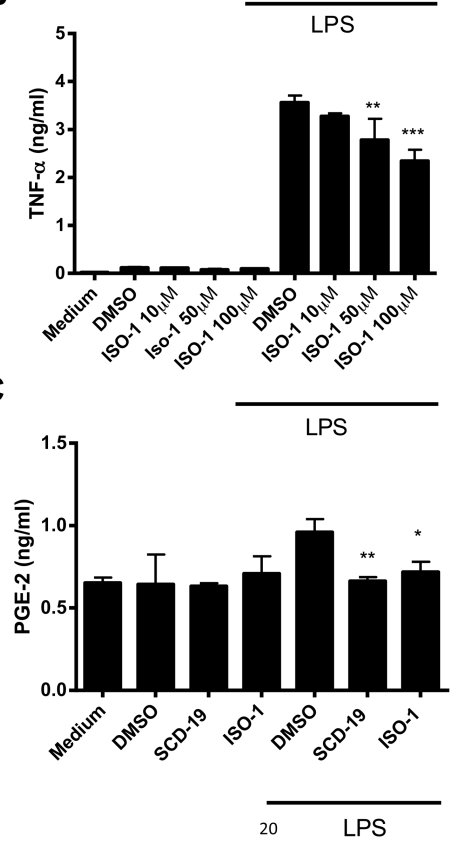

Figure 2. SCD-19 inhibits the LPS-associated release of TNF $\alpha$ and $P G E_{2^{\prime}}$ (A) RAW macrophage cells were plated at $1 \times 10^{5}$ per well and pretreated with SCD-19 at 10,50 or $100 \mu \mathrm{mol} / \mathrm{L}$ or DMSO for $30 \mathrm{~min}$ before stimulation with $100 \mathrm{ng} / \mathrm{mL}$ LPS. After $16 \mathrm{~h}$, supernatants were collected and assessed for levels of TNF $\alpha$ by ELISA. Data are presented as mean and $S D(n=6)$. (B) RAW macrophage cells were plated at $1 \times 10^{5}$ per well and pretreated with $1 \mathrm{SO}-1$ at 1050 or $100 \mu \mathrm{mol} / \mathrm{L}$ or DMSO for 30 min before stimulation with $100 \mathrm{ng} / \mathrm{mL}$ LPS. After $16 \mathrm{~h}$, supernatants were collected and assessed for levels of TNF $\alpha$ by ELISA. Data are presented as mean and SD $(n=6)$. (C) RAW 264.7 macrophage cells were plated at a cell density at $1 \times 10^{5} \mathrm{per}$ well and were pretreated with $100 \mu \mathrm{mol} / \mathrm{L}$ SCD-19 or ISO-1 for 30 min before the addition of $100 \mathrm{ng} / \mathrm{mL}$ LPS. After $16 \mathrm{~h}$, cell culture supernatants were collected. Supernatants were assessed using a PGE $E_{2}$ ELISA kit. ${ }^{*} p<$ $0.05,{ }^{* *} p<0.01,{ }^{* * *} p<0.001$. Data presented as mean $\pm S D(n=6)$.

\section{SCD-19 Inhibits MIF-Induced Rate of} Cell Growth of LLC Cells In Vitro

MIF has been found to be expressed in a number of tumor types including lung cancer. MIF has previously been shown to directly promote cellular proliferation of LLC cells (20). We examined whether inhibition of the tautomerase activity of endogenous MIF or that of extracellular added recombinant MIF (100 ng/mL) by SCD-19 would inhibit rate of cell growth in vitro. In both cases, SCD-19 exceeded the effect of ISO-1 and significantly reduced rate of cell growth of the lung cancer cell line. A total of $100 \mu \mathrm{mol} / \mathrm{L}$ SCD19 inhibited both basal tumor (SCD-19, $47 \%$ reduction $\pm 0.33,{ }^{* * *} p<0.001$; ISO- 1 $8 \%$ reduction \pm 0.31 ) and extracellular added MIF rate of cell growth (SCD-19, $48 \%$ reduction $\pm 0.326,{ }^{* *} p<0.01$; ISO- 1 , $11 \%$ reduction \pm 0.952 ) (Figures $3 \mathrm{~A}, \mathrm{~B}$ ).

\section{MIF Supports Tumor Growth In Vivo}

Before assessing the effectiveness of SCD-19 in LLC growth in vivo, we first confirmed the importance of functioning host-derived MIF in the setting of LLC cell primary tumor growth. Subcutaneous LLC tumors were established in Mif-KO and $M i f^{P 1 G}$ as well as wild-type (WT) control mice. Tumor growth was substantially reduced in both our Mif-KO mice $\left(62 \%\right.$ reduction, $\left.{ }^{* *} p<0.01\right)$ and $M i f^{P 1 G}$ transgenic mice $\left(42 \%\right.$ reduction, $\left.{ }^{*} p<0.05\right)$ compared with WT mice (Figure 4A).

\section{Inhibition of MIF by SCD-19 Reduced Lung Tumor Growth In Vivo}

Having established that MIF is important for LLC growth and that SCD-19 could specifically inhibit MIF tautomerase enzymatic activity, we then assessed the potential for use of SCD-19 in vivo. By using a subcutaneous murine model of LLC, we treated mice twice weekly intraperitoneally with $35 \mathrm{mg} / \mathrm{kg}$ SCD-19, ISO-1 or vehicle (control) starting on the day of tumor inoculation. Mice receiving SCD-19 were found to have significantly reduced tumor volume (90\% reduction, $\left.{ }^{* * *} p<0.001\right)$ compared with control and vehicle-treated mice, whereas mice receiving ISO-1 had a less
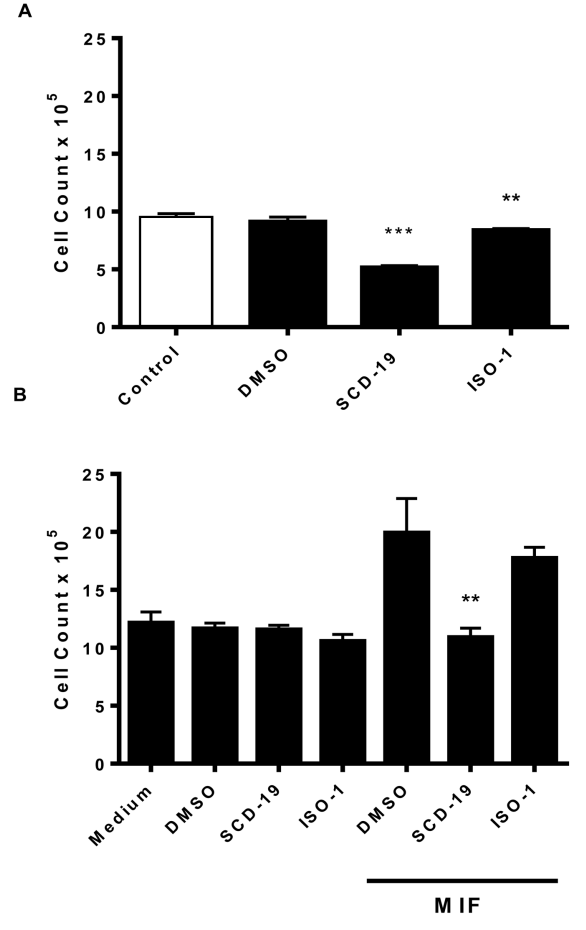

Figure 3. MIF enzymatic activity promotes rate of cell growth and tumor growth. (A) SCD-19 significantly reduces endogenous LLC growth rate. LLC cells were plated at $5 \times 10^{4}$ cells $/ \mathrm{mL}$ and then pretreated with SCD-19 and ISO-1 at a concentration of $100 \mu \mathrm{mol} / \mathrm{L}$. The cells were incubated at $37^{\circ} \mathrm{C}$ in a humidified $5 \% \mathrm{CO}_{2}$ incubator, and the cells were harvested using centrifugation after $48 \mathrm{~h}$ and counted by using a hemocytometer $\left({ }^{* * *} p<0.001\right.$, ${ }^{* *} p<0.01$; data presented as mean $\pm S D$, $n=3)$. (B) LLC cells were plated at a cell density of $5 \times 10^{4}$ cells $/ \mathrm{mL}$ and pretreated with $100 \mu \mathrm{mol} / \mathrm{L}$ of SCD-19 or ISO-1. Cells were subsequently treated with $100 \mathrm{ng} / \mathrm{mL}$ recombinant MIF and incubated at $37^{\circ} \mathrm{C}$ in a humidified $5 \% \mathrm{CO}_{2}$ incubator. After $48 \mathrm{~h}$, cells were harvested by centrifugation and counted by using a hemocytometer ( ${ }^{* *} p<0.01$; data presented as mean $\pm S D, n=6$ ).

pronounced effect $\left(60 \%\right.$ reduction, ${ }^{* *} p<$ 0.01 ) (Figure 4A). Having confirmed that SCD-19 could inhibit tumor growth when administered prophylactically, we decided to replicate better the human clinical situation and only administer our inhibitor when the tumor was palpable on the flank of the mouse (d 7 after 


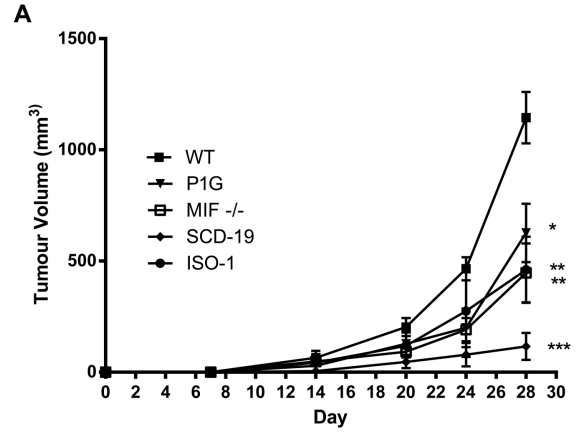

B

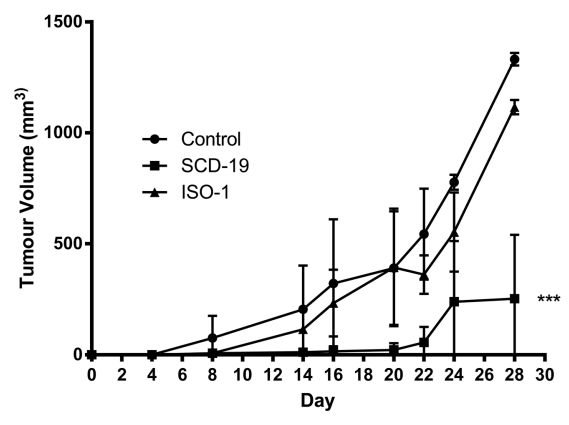

Figure 4. LLC tumor volume is significantly reduced in Mif-KO and Mif ${ }^{P I G}$ mice compared with WT, and SCD-19 reduces tumor growth when given prophylactically and therapeutically. (A) LLC cells were cultured until $80 \%$ confluency. Animals were shaved with an electric shaver $24 \mathrm{~h}$ before subcutaneous injection. LLC cells $\left(5 \times 10^{5}\right)$ were injected subcutaneously into the left flank of C57BL/6 WT mice and Mif ${ }^{P I G}$ and MIF-KO mice (6-8 wks). Animals were injected intraperitoneally 30 min before tumor inoculation and twice weekly thereafter, with 35 mg/kg SCD-19 or ISO-1 as indicated.

Animals were monitored for $28 \mathrm{~d}$. Calliper measurements were taken twice weekly, and tumor volume $\left(\mathrm{mm}^{3}\right)$ was calculated as $0.5 \times L \times W^{2}\left({ }^{*} p<0.05,{ }^{* *} p<0.01,{ }^{* * *} p<\right.$ $0.001, n=4)$. (B) LLC cells $\left(5 \times 10^{5}\right)$ were injected subcutaneously into the left flank of C57BL/6 mice. Once the tumor was pal-

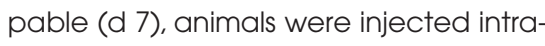
peritoneally with $35 \mathrm{mg} / \mathrm{kg}$ SCD-19, ISO-1 or DMSO and twice weekly thereafter. Animals were monitored for $28 \mathrm{~d}$. Calliper measurements were taken twice weekly, and tumor volume $\left(\mathrm{mm}^{3}\right)$ was calculated as $0.5 \times L \times W^{2}\left({ }^{* * *} p<0.001, n=4\right)$.

tumor inoculation). Again, mice were treated twice weekly with $35 \mathrm{mg} / \mathrm{kg}$ SCD-19, ISO-1 or vehicle intraperi- toneally and tumor growth was assessed. SCD-19 again significantly reduced tumor growth $(81 \%$ reduction in tumor, $\left.{ }^{* * *} p<0.001\right)$ compared with ISO-1, which did not significantly reduce tumor growth (16\% reduction) (Figure 4B).

\section{DISCUSSION}

It has long been recognized that chronic inflammatory states have the capacity for in situ malignant transformation. MIF, a proinflammatory cytokine, has been highlighted as a potential driver of this malignant transformation (11). In the context of lung cancer, MIF expression has been shown to correlate with prognosis, and it has been suggested as a potential biomarker in non-small-cell lung cancer (28). An in vivo model of LLC set in an MIF overexpressing environment showed that MIF directly enhanced tumor growth (20). Expression of MIF and its receptor CD74 has been shown to correlate with increased angiogenic factor secretion in lung cancer cell lines $(18,29)$. In vitro studies have shown that global inhibition of functional MIF in lung cancer cell lines is an effective way to inhibit many of the hallmarks of tumor development such as angiogenesis and metastasis (19).

The enzymatic function of MIF has been previously shown to be required for optimal signaling in inflammatory and tumor growth pathways. Mutation of the tautomerase active site or inhibition by chemical inhibitors has previously been shown to attenuate key processes required for tumor growth, namely MIFinduced ERK phosphorylation, COX-2 induction and p53 inhibition.

The P1G transgenic mouse expresses a mutated MIF resulting in a loss of enzymatic activity and in turn loss of specific biological activity. Using this in vivo Mif $^{\text {P1G }}$ model, authors have previously looked at the enzymatic activity of MIF with regard to tumor progression, benzo[ $\alpha]$ pyrene was used to chemically induce skin tumors in the Mif ${ }^{P 1 G}$ mice (30). Significantly fewer tumors were induced compared with WT MIF mice. In a model of colorectal cancer, increased levels of MIF expression were shown to correlate with both tumor differentiation and also in lymph node and liver metastasis. Using the MIF inhibitor ISO-1 to therapeutically treat the mice resulted in a significant reduction in tumor volume, decreased angiogenesis and also lower levels of liver metastasis (13).

Recent published work has identified two additional tumor-associated pathways where MIF plays an important role. AMP-activated protein kinase (AMPK) is an enzyme that regulates cellular metabolism in response to stress and is activated by the tumor suppressor liver kinase B1 (LKB1). MIF has been shown to deactivate AMPK enzyme supporting tumor growth in non-small-cell lung cancer cells (31). MIF has also been shown to mediate alternative activation of tumor-associated macrophages in a melanoma model. In both macrophages from MIF null mice and mice treated with the tautomerase inhibitor 4-IPP, immunosuppression was inhibited, which lead to reduced tumor growth and increased survival (32).

Based on this knowledge, we believe that targeting the hydrophobic pocket of MIF, the location of the tautomerase enzymatic active site of MIF, represents a valid anticancer therapeutic target.

Small-molecular-weight inhibitors of the tautomerase enzymatic activity of MIF have been developed previously. ISO-1 represents one of the best characterized of these specific inhibitors and has been analyzed in a number of tumor models. In a model of prostate cancer, inhibition of MIF by ISO-1 significantly attenuated tumor growth and angiogenesis (33). Similarly, in a model of colon cancer, ISO-1 was capable of significantly reducing tumor mass (13). However, to date, the use of small molecule inhibitors targeting the tautomerase enzymatic activity of MIF has not been described in lung cancer. Small molecule inhibitors have the advantage (over antibodies) of ease of uptake by cells and the potential of exerting their inhibitory capacity both intracellularly and extracellularly. 
Through the protein-ligand docking program FRED, we identified a number of putative small molecule inhibitors of MIF. SCD-19 was taken through from in vitro analysis to an in vivo model of lung cancer. To assess the specificity of SCD19 , we used a cell-free assay consisting of recombinant MIF and the substrate L-dopachrome methyl ester. Complete inhibition of MIF-dependent tautomerization of the substrate was achieved by using a concentration of $100 \mu \mathrm{mol} / \mathrm{L}$. A Ki value of $32 \mu \mathrm{mol} / \mathrm{L}$ was calculated that compares favorably to that of ISO-1, the most commonly used inhibitor of MIF enzymatic activity. We also analyzed our compound using an LDH assay to assess potential cellular toxicity of our compound. We analyzed LDH release from collected supernatants derived from our previous experiments. This step allowed us to see that our compound was not toxic to the cells, causing cell death.

MIF promotes development of an inflammatory environment through promotion of TNF $\alpha$ and COX-2 production. These activities are also associated with the induction and promotion of tumors. To further characterize SCD-19, we determined whether this inhibitor could attenuate MIF-associated inflammatory signals. LPS-induced production of TNF $\alpha$ was significantly decreased when the cells were preincubated with SCD19. Furthermore, COX-2-dependent $\mathrm{PGE}_{2}$ in RAW macrophage cells was attenuated to control levels after pre-incubation with SCD-19. MIF has been shown by other authors to upregulate COX-2 and, in turn, $\mathrm{PGE}_{2}$ production (16). Previously, a study looking at rheumatoid arthritis showed that treating fibroblast-like synoviocytes with recombinant MIF significantly increased the amount of COX-2 activity and in turn the amount of $\mathrm{PGE}_{2}$ production in the cells (34). Reports have shown that upregulation of COX-2 and $\mathrm{PGE}_{2}$ leads to tumor growth and metastasis of tumors, since $\mathrm{PGE}_{2}$ promotes angiogenesis (35). Because SCD-19 reduces the production of $\mathrm{PGE}_{2}$ from RAW 264.7 macro- phages, reduced tumor vascularization and thus volume results. Th1 responses are required to attenuate tumor growth (36). Because SCD-19 reduces TNF $\alpha$ production in RAW 264.7 macrophages, a decrease in tumor incidence and also tumor volume may result.

It has previously been shown that addition of recombinant MIF to LCC cells directly promotes cell growth rate. Before taking SCD-19 into the in vivo model of LLC, we established the important role for the tautomerase activity of MIF for the growth and proliferation of these particular tumor cells. SCD-19 has the ability both to inhibit MIF-induced cell growth rate of these cancer cells and also to inhibit basal cell growth rate, presumable via inhibition of endogenous tumor-derived MIF. In vivo we compared growth of the LLC cell line in WT mice versus Mif-KO mice and Mif ${ }^{P 1 G}$ mice expressing the tautomerase-null MIF protein. The important role that blocking the hydrophobic active site of the tautomerase enzymatic activity of MIF plays in promoting tumor growth is evident from both in vitro and in vivo work. A dosing strategy was used similarly to previously published work with enzymatic inhibitors (22). Mice were initially treated with tumor inoculation. Animals were then administered with SCD-19 twice weekly. Significant tumor volume reduction in treated animals was found. To mimic a more clinically relevant setting, we then treated mice posttumor inoculation, waiting until the tumor was palpable before commencing treatment. At this point ( $7 \mathrm{~d}$ after tumor inoculation) mice were treated twice weekly as before. Primary tumor volume was found to be significantly reduced $(>80 \%)$ in treated animals. The results presented here highlight the importance of this tautomerase activity in driving cancer growth and provide evidence for targeting this unique activity as part of an anticancer drug discovery platform. When comparing inhibition of tumor growth in both our Mif-KO and knock-in mice experiments to WT mice treated with our novel inhibitors, we found sig- nificantly enhanced inhibition in our treated mice.

\section{CONCLUSION}

Recent studies have focused on the MIF produced by the tumor stroma and not the tumor cells themselves. There is a lot of current research surrounding the role of stromal-cell MIF in tumor growth. Simpson et al. (37) looked at the role of MIF in tumor growth. They looked at a metastatic breast cancer model and demonstrated that tumor-derived MIF has the ability to promote tumor growth through inflammatory cells within the tumor. In this study, MIF was seen to increase the number of myeloid- derived suppressor cells (MDSCs) within the tumor. Using an inhibitor of MIF, they demonstrated a reduction in MDSCs within the tumor that is similar to a reduction of MIF. While this study demonstrates that the stromal MIF is important, it also highlights the importance of therapeutically inhibiting MIF with chemical inhibitors.

In this model, both tumor- and hostderived MIF are targeted compared with our transgenic/knock-in models, where LLC cells still have the capacity to produce endogenous-active MIF within the tumor microenvironment. To maximize cancer inhibition, our data clearly show that a strategy that targets both hostand tumor-derived MIF is significantly more efficacious. In summary, we have shown that targeting the unique tautomerase enzymatic activity of MIF, using our novel inhibitor, represents a valid strategy attenuating lung cancer growth.

\section{ACKNOWLEDGMENTS}

SC Donnelly was supported by grants from Science Foundation Ireland (SFI), the Health Research Board (HRB) and the Irish Lung Foundation (ILF). R Bucala was supported by National Institutes of Health Grant AI042310. G Fingerle-Rowson was supported by the Jose Carreras Leukaemia Foundation (Grant R06/20) and by the German Research Council (Grant 712/2-2). 


\section{DISCLOSURE}

The authors declare that they have no competing interests as defined by Molecular Medicine, or other interests that might be perceived to influence the results and discussion reported in this paper.

\section{REFERENCES}

1. Ferlay J, et al. (2010) Estimates of worldwide burden of cancer in 2008: GLOBOCAN 2008. Int. J. Cancer. 127:2893-917.

2. American Cancer Society. (c2012) Cancer Facts $\mathcal{E}$ Figures 2012 [PDF on the Internet]. Atlanta: American Cancer Society; 2012; [cited 2015 Mar 25]. Available from: http://www.cancer.org/ research/cancerfactsfigures/cancerfactsfigures/ cancer-facts-figures-2012

3. Donnelly SC, et al. (1997) Regulatory role for macrophage migration inhibitory factor in acute respiratory distress syndrome. Nat. Med. 3:320-3.

4. Rossi AG, et al. (1998) Human circulating eosinophils secrete macrophage migration inhibitory factor (MIF): potential role in asthma. J. Clin. Invest. 101:2869-74

5. Adamali $\mathrm{H}$, et al. (2012) Macrophage migration inhibitory factor enzymatic activity, lung inflammation, and cystic fibrosis. Am. J. Respir. Crit. Care Med. 186:162-9.

6. Plant BJ, et al. (2005) Cystic fibrosis, disease severity, and a macrophage migration inhibitory factor polymorphism. Am. J. Respir. Crit. Care Med. 172:1412-5.

7. Calandra T, Bernhagen J, Mitchell RA, Bucala R. (1994) The macrophage is an important and previously unrecognized source of macrophage migration inhibitory factor. J. Exp. Med. 179:1895-902.

8. Bacher M. (1996) An essential regulatory role for macrophage migration inhibitory factor in T-cell activation. Proc. Natl. Acad. Sci. U. S. A. 93:7849-54.

9. Daryadel A, Grifone RF, Simon HU, Yousefi S. (2006) Apoptotic neutrophils release macrophage migration inhibitory factor upon stimulation with tumour necrosis factor-alpha. J. Biol. Chem. 281:27653-61.

10. Conroy H, Mawhinney L, Donnelly SC. (2010) Inflammation and cancer: macrophage migration inhibitory factor (MIF): the potential missing link. QJM. 103:831-6.

11. Bucala R, Donnelly SC. (2007) Macrophage migration inhibitory factor: a probable link between inflammation and cancer. Immunity. 26:281-5.

12. Meyer-Siegler KL, et al. (2007) Macrophage migration inhibitory factor (MIF) gene polymorphisms are associated with increased prostate cancer incidence. Genes Immun. 8:646-52.

13. He XX, et al. (2009) Macrophage migration inhibitory factor promotes colorectal cancer. Mol. Med. 15:1-10.

14. Ren Y, et al. (2003) Macrophage migration inhibitory factor: roles in regulating tumour cell migration and expression of angiogenic factors in hepatocellular carcinoma. Int. J. Cancer. 107:22-9.
15. Tomiyasu M, Yoshino I, Suemitsu R, Okamoto T, Sugimachi K (2002). Quantification of macrophage migration inhibitory factor mRNA expression in non-small cell lung cancer tissues and its clinical significance. Clin. Cancer Res. 8:3755-60.

16. Mitchell RA, et al. (2002) Macrophage migration inhibitory factor (MIF) sustains macrophage proinflammatory function by inhibiting p53: regulatory role in the innate immune response. Proc. Natl. Acad. Sci. U. S. A. 99:345-50.

17. Winner M, Koong AC, Rendon BE, Zundel W, Mitchell RA. (2007) Amplification of tumour hypoxic responses by macrophage migration inhibitory factor-dependent hypoxia-inducible factor stabilization. Cancer Res. 67:186-93.

18. White ES, et al. (2003) Macrophage migration inhibitory factor and CXC chemokine expression in non-small cell lung cancer: role in angiogenesis and prognosis. Clin. Cancer Res. 9:853-60.

19. Rendon BE, et al. (2007) Regulation of human lung adenocarcinoma cell migration and invasion by macrophage migration inhibitory factor. J. Biol. Chem. 282:29910-8

20. Arenberg D, et al. (2010) Macrophage migration inhibitory factor promotes tumour growth in the context of lung injury and repair. Am. J. Respir. Crit. Care Med. 182:1030-7.

21. Rosengren E, et al. (1996) The immunoregulatory mediator macrophage migration inhibitory factor (MIF) catalyzes a tautomerization reaction. Mol. Med. 2:143-9.

22. Al-Abed Y, et al. (2005) ISO-1 binding to the tautomerase active site of MIF inhibits its proinflammatory activity and increases survival in severe sepsis. J. Biol. Chem. 280:36541-4.

23. Palud A, et al. (2013) Macrophage migration inhibitory factor inhibition is deleterious for highfat diet-induced cardiac dysfunction. PLoS One. 8:e58718.

24. Fingerle-Rowson G, et al. (2009) A tautomerasenull macrophage migration-inhibitory factor (MIF) gene knock-in mouse model reveals that protein interactions and not enzymatic activity mediate MIF-dependent growth regulation. Mol. Cell. Biol. 29:1922-32.

25. Winner M, et al. (2008) A novel, macrophage migration inhibitory factor suicide substrate inhibits motility and growth of lung cancer cells. Cancer Res. 68:7253-7.

26. Cooke G, Armstrong ME, Donnelly SC. (2009) Macrophage migration inhibitory factor (MIF), enzymatic activity and the inflammatory response. BioFactors. 35:165-8.

27. Jemal A, Siegel R, Xu J, Ward E. (2010) Cancer statistics, 2010. CA Cancer J. Clin. 60:277-300.

28. Gamez-Pozo A, et al. (2012) PTRF/cavin-1 and MIF proteins are identified as non-small cell lung cancer biomarkers by label-free proteomics. PLoS One. 7:e33752.

29. McClelland M, Zhao L, Carskadon S, Arenberg D. (2009) Expression of CD74, the receptor for macrophage migration inhibitory factor, in nonsmall cell lung cancer. Am. J. Path. 174:638-46.
30. Fingerle-Rowson G, et al. (2003) The p53-dependent effects of macrophage migration inhibitory factor revealed by gene targeting. Proc. Natl. Acad. Sci. U. S. A. 100:9354-9.

31. Brock SE, Rendon BE, Yaddanapudi K, Mitchell RA. (2012) Negative regulation of AMP-activated protein kinase (AMPK) activity by macrophage migration inhibitory factor (MIF) family members in non-small cell lung carcinomas. J. Biol. Chem. 287:37917-25.

32. Yaddanapudi K, et al. (2013) Control of tumourassociated macrophage alternative activation by macrophage migration inhibitory factor. J. Immunol. 190:2984-93.

33. Meyer-Siegler KL, Iczkowski KA, Leng L, Bucala R, Vera PL. (2006) Inhibition of macrophage migration inhibitory factor or its receptor (CD74) attenuates growth and invasion of DU-145 prostate cancer cells. J. Immunol. 177:8730-9.

34. Sampey AV, Hall PH, Mitchell RA, Metz CN, Morand EF. (2001) Regulation of synoviocyte phospholipase A2 and cyclooxygenase 2 by macrophage migration inhibitory factor. Arthritis Rheum. 44:1273-80.

35. Liu M, et al. (2007) EGFR signaling is required for TGF-beta 1 mediated COX-2 induction in human bronchial epithelial cells. Am. J. Respir. Cell Mol. Biol. 37:578-88.

36. Jones EA, Pringle JH, Angel CA, Rees RC. (2002) Th1/Th2 cytokine expression and its relationship with tumour growth in B cell non-Hodgkin's lymphoma (NHL). Leuk. Lymphoma. 43:1313-21.

37. Simpson KD, Templeton DJ, Cross JV. (2012) Macrophage migration inhibitory factor promotes tumour growth and metastasis by inducing myeloid-derived suppressor cells in the tumour microenvironment. J. Immunol. 189:5533-40. 